\title{
Non-infectious status indicated by detectable $\lg G$ antibody to SARS-CoV-2
}

\author{
David W. Denning, ${ }^{* 1,2}$ Anthony Kilcoyne ${ }^{2,3}$ and Cemal Ucer ${ }^{4,5}$
}

\section{Key points}

SARS-CoV-2 viral infectivity lasts for eight days from the start of infection.
SARS-CoV-2 IgG antibody is usually neutralising, is first detectable at 11 days after infection and persists for months.
People with detectable SARS-CoV-2 IgG antibody can safely be regarded as non-infectious ( $>99 \%$ level of certainty).

\begin{abstract}
A key tenet of protection from infection for dentists is to know who is not infectious. The evidence base regarding protection from respiratory pathogens in dentistry is poor. Those with a positive SARS-CoV-2 IgG antibody are noninfectious ( $>99 \%$ certainty) and can be safely treated with good universal precautions, even for aerosol generating procedures. Viral infectivity with SARS-CoV-2 lasts eight days, unlike viral polymerase chain reaction (PCR) swab tests which can persist for as long as seven weeks. SARS-CoV-2 IgG antibody becomes detectable from 11 days after infection. SARS-CoV-2 IgG antibodies are usually neutralising against the virus and their direct antiviral activity was partially demonstrated in 33,000 patients with COVID-19 treated with convalescent plasma in the USA. So, a positive SARS-CoV-2 IgG antibody is a much more accurate determination of infectiousness than a repeat PCR which is only 70\% sensitive. It remains to be seen whether SARS-Cov-2 vaccine responses include protective IgG titres and, once vaccines become widespread, can be used to assist decision-making on appropriate personal protective equipment (PPE) in dentistry.
\end{abstract}

\section{Introduction}

It is helpful for dentists (and other community healthcare professionals) to know who is not infectious for any pathogen, but especially highly transmissible ones such as SARSCoV-2. Extraordinarily few studies have addressed the risk to dental professionals of respiratory viruses, ${ }^{1}$ and the efficacy of universal precautions (standard personal protective equipment $[\mathrm{PPE}]$ ) versus various means of enhancing protection is not known. What is clear is that extra PPE precautions

'Division of Infection, Immunity and Respiratory Medicine, Faculty of Biology, Medicine and Health, Manchester Academic Health Science Centre, University of Manchester Manchester, UK; ${ }^{2}$ DenScreen Ltd, 86-90 Paul Street, London, EC2A 4NE, UK; ${ }^{3}$ The SmileSpecialist Centre, 9-13 Station Road, Haworth, Keighley, West Yorkshire, BD22 $8 \mathrm{NL}$, UK; ${ }^{4}$ Postgraduate Dental Studies, School of Health and Society, University of Salford, Greater Manchester, UK; ${ }^{5}$ ICE Postgraduate Dental Institute \& Hospital, Salford Quays, Salford, M50 3XZ, UK

*Correspondence to: David Denning

Email address: ddenning@manchester.ac.uk

Refereed Paper.

Accepted 23 September 2020

https://doi.org/10.1038/s41415-020-2228-9 are unnecessary if the patient is known to be non-infectious.

The immune response to SARS-CoV-2 is complex and changes during and after infection. Here, we describe the current state of knowledge regarding viral infectiousness, molecular (polymerase chain reaction [PCR] swab) testing, antibody production, clinical interpretation and immunity. We summarise the implications for dental practice.

SARS-CoV-2 is the name of the coronavirus causing the symptomatic disease COVID-19; those with asymptomatic infection are best referred to as having SARS-CoV-2 infection.

\section{The course of SARS-CoV-2 infection}

After infection, SARS-CoV-2 viral load rises to maximum levels about two days before symptoms start and remains high for five days, then falls after 7-8 days ${ }^{2,3,4}$ (Fig. 1). Live virus is not culturable after eight days in non-immunocompromised people (work in Germany, Canada and France), 2,3,4 consistent with transmission ceasing 6-8 days after symptom onset. ${ }^{5,6}$ Viral RNA remains detectable in mouth and nasal swabs, sputum and faeces for up to seven weeks, ${ }^{2,7}$ but it represents non-viable remnants of virus. Asymptomatic infected people probably have the same viral trajectory, with slightly less virus on average. The amount of virus detectable in a given person on any given day varies enormously from around 100 virions to over $10,000,000,000$ per sample. ${ }^{8}$

There is a single report of a heart transplant patient still growing the virus at five weeks after infection. ${ }^{9}$ So, immunocompromised individuals may excrete the virus for much longer and this key point in a medical history should be checked.

\section{The antibody response to SARS- CoV-2}

The earliest antibody to appear in blood is SARS-CoV-2 immunoglobulin M (IgM), usually from day 5-7 after symptoms appear, but sometimes later. ${ }^{2,10,11,12,13}$ Not all patients produce IgM and the amount of IgM in blood rises rapidly and falls away earlier than immunoglobulin G (IgG). IgM antibody 
primarily functions as defence against gram-negative bacteria, but is a marker of infection against most pathogens. IgG and immunoglobulin A (IgA) production follows IgM, with these antibodies detectable from day 11 and reaching a maximum 3-4 weeks after infection. ${ }^{2,10,11,12,13}$ IgG and IgA antibody titres are highly correlated. ${ }^{10}$ IgA antibody protects mucosal surfaces from infection. Not all those with COVID-19 produce IgG or IgA.

Antibodies are directed against several viral proteins: the surface spike protein (a glycoprotein), nucleocapsid phosphoprotein, membrane glycoprotein, envelope protein and several other non-structural proteins. ${ }^{14}$ Most antibody tests use spike and/or nucleocapsid protein fragments as capture antigens. Some antigen preparations used in these antibody tests have more antibody affinity than others.

The best blood and finger-prick SARSCoV-2 antibody tests have about 95\% sensitivity for IgG (1/20 genuine cases are missed) in patients with COVID-19 and a specificity of about $99 \%$ (1/100 false positive cases). ${ }^{15,16,17}$ These 'false positive' tests probably represent residual immunity for other coronavirus infection, with cross reactions. ${ }^{12}$ This high level of performance of antibody testing is much better than the SARS-CoV-2 swab tests for viral RNA, which are about $70 \%$ sensitive. ${ }^{18}$ Most asymptomatic SARS-CoV-2 infections (about $70 \%$ of the total) also usually generate an IgG antibody response, but not all. ${ }^{10,13}$ At least some of those without a detectable IgG response have a detectable neutralising antibody, clearly directed to viral proteins not used in the IgG antibody assay. ${ }^{13}$

\section{Other key elements of protection}

SARS-CoV-2 IgG antibody is usually a neutralising antibody. ${ }^{13,17,19}$ Convalescent plasma therapy (in which these antibodies are the primary antiviral effector) has been extensively used in the USA and other countries, and one randomised trial demonstrated a reduction in COVID-19 mortality from $40 \%$ to $5 \%(\mathrm{p}=0.03) .{ }^{20} \mathrm{~A}$ fixed-effects analysis of all randomised and match control series indicates a reduction in mortality from $22 \%$ to $10 \%$ with convalescent plasma therapy. ${ }^{21}$ Therefore, the IgG antibody is a critical component of defence against SARS-CoV-2 infection. Alongside the IgG antibody is the IgA antibody and cytotoxic

Fig. 1 Summary of SARS-CoV-2 viral and PCR kinetics over time, compared with the $\operatorname{lgM}$ and IgG responses. There is much individual variation, but viable virus falls to zero in 7-10 days in all patients, apart from those who are immunocompromised. Image courtesy of DenScreen

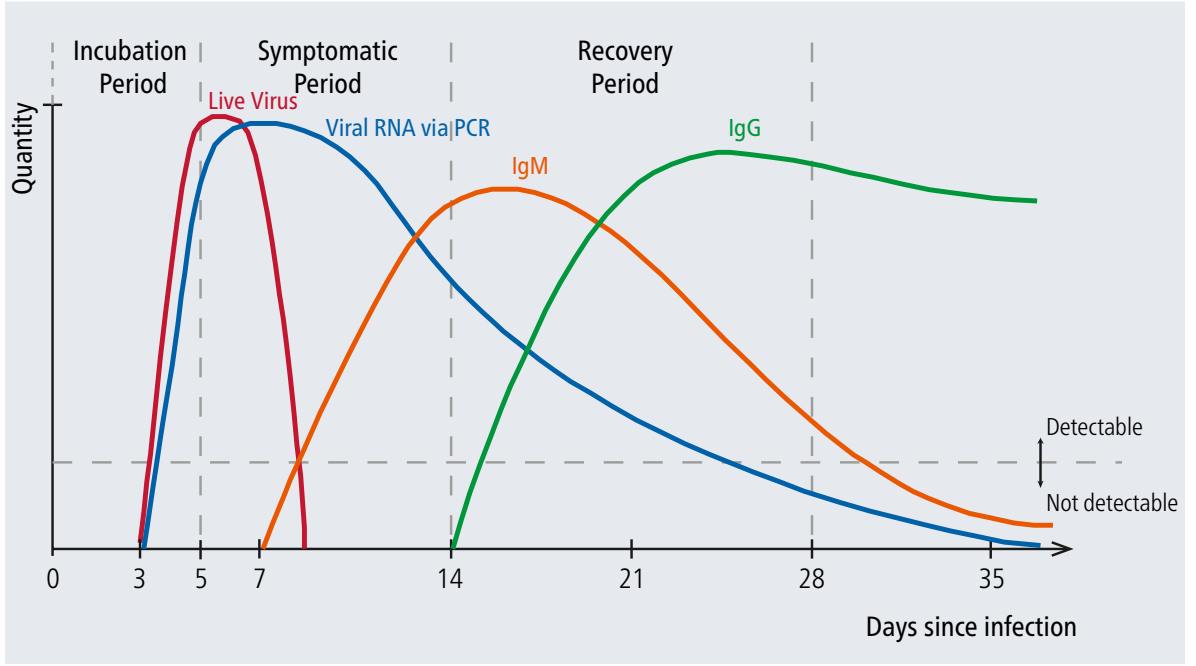

T cells directed against the SARS-CoV-2 virus. ${ }^{10,13}$ Immunity against reinfection is now demonstrated in a monkey model, ${ }^{22}$ with a neutralising antibody clearly being a key component of the immune response, orchestrated by T-helper and memory cells. ${ }^{2,10,22,23}$

\section{The value of rapid antibody testing in determining infectiousness}

How can we be confident that a person is not infectious? Firstly, those who have had documented COVID-19 will have had protective immune responses and cleared the virus (confirmed test results with compatible illness are convincing). Secondly, those asymptomatic patients who were in contact with an infected person will have at least short-lived immunity - weeks or months, but probably not years (needs further longitudinal research). Thirdly, those who have never been in contact with a person or objects carrying SARS-CoV-2 will be non-infectious (difficult to ascertain, but might include some very strict self-isolators).

To what extent can simple antibody testing assist in addressing the first and second points above? Given that there is no overlap in time between the end of viral infectiousness and the earliest formation of IgG antibody, if specific antiviral IgG is detectable, that person is extremely unlikely to be infectious. As IgG antibodies are then detectable for weeks or months, noninfectiousness can be assured for at least as long as these antibodies are detectable, and probably a lot longer because of residual T cell memory.

Antibody levels do fall over time. ${ }^{9,24}$ The length of time that IgG antibodies remain detectable after COVID-19 varies from as short as a few weeks in some asymptomatic infections to many months. Once IgG has been detected, it is highly likely that the person is partially or fully immune for at least months.

\section{Antigen testing}

Antigen point-of-care testing is now also possible and takes about 20 minutes. Nasopharyngeal and mouth swabs are the usual sample types in clinical evaluations, but saliva may be as useful. Several tests are marketed, but none are applied to dentistry as yet. Antigen testing is likely to be slightly less sensitive than $\mathrm{PCR}^{25}$ and so may be a better indicator of infectiousness. ${ }^{26}$

\section{Implications for dentistry}

Those with a positive SARS-CoV-2 IgG test are non-infectious ( $>99 \%$ certainty). The best finger-prick antibody tests take ten minutes and such diagnostic assessment is well within the official and permitted scope of practice of dentists (as the Care Quality Commission have directly indicated in their regular bulletins).

The implications for dentistry can be logically summarised as follows: 


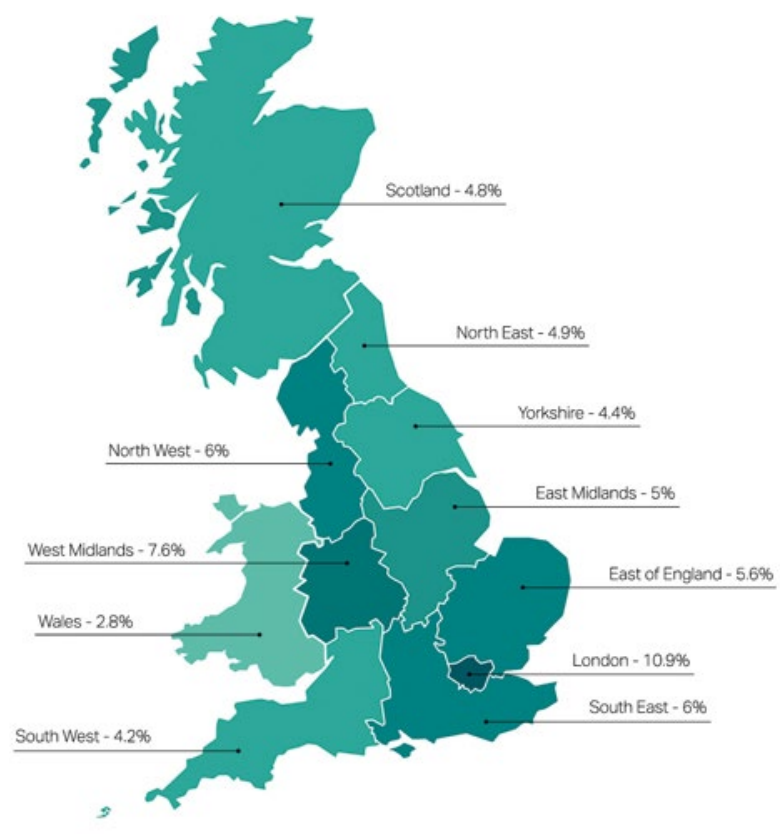

Fig. 2 SARS-CoV-2 antibody prevalence across the UK based on the UK Biobank SARS-CoV-2 Serology Study (27 May-6 July 2020), the REACT study (20 June-13 July 2020) and the population-based seroprevalence surveillance for Scotland (April-21 June 2020). Image courtesy of DenScreen

1. SARS-CoV-2 IgG antibody is directly linked to non-infectiousness

2. Non-infectious people need good universal PPE precautions, but not extra enhanced precautions for aerosol generating procedures

3. IgG antibody fades over time, so the earlier after infection testing is done, the more likely they are to be positive - with helpful documentation of recent SARSCoV-2 infection

4. Vulnerable people who are currently denied access to routine/urgent dental treatment can be safely treated if shown to be non-infectious

5. The longitudinal patient relationship in dentistry lends itself naturally to antibody testing and retesting, as well as for other services such as vaccine delivery, health screening and wider prevention services.

It is also likely that assessment of continuing vaccine protection can be assessed with IgG antibody tests, and a neutralising IgG antibody is a key marker of vaccine responses, aligned with other immune parameters such as $\mathrm{T}$ cell responses.

We need to use all the tools at our disposal for controlling SARS-CoV-2. One key tool is IgG antibody testing, which is helpful when positive and less helpful when negative alone, but potentially powerful when combined with other approaches, given no other single aspect can deliver $100 \%$ surety by itself. Several comparative antibody studies are now published, and the reliability and diagnostic performance of testing in hospital laboratories and finger-prick tests in ambulatory settings are indistinguishable. As the number of people infected who become non-infectious will greatly increase, this can be reliably monitored with $\operatorname{IgG}$ antibody testing and monitored for wider public health good.

The population prevalence of SARS-CoV-2 varies in the UK, from high in London to low in Wales (Fig. 2).27,28,29 Therefore, the costeffectiveness and utility of antibody testing varies, but this does not negate the value of IgG antibody detection going forwards for those who are keen to know if they have had SARSCoV-2 infection (and therefore unlikely to be a source of infection for months or years) and in infection control.

It has been disappointing to see much poor science reporting on SARS-CoV-2 antibody testing and an apparent obsession with ' $100 \%$ accuracy' (which is unattainable with this virus), which has actually obscured the real value of antibody testing in many situations, including in dentistry.
Conflict of interest

DWD and $A K$ are co-founders of $w w w . D e n S c r e e n$. co.uk, which assists dentists with working safely in the COVID-19 era.

\section{Acknowledgements}

DWD is partially supported by the NIHR

Biomedical Research Centre in Manchester.

\section{References}

1. Davies K J, Herbert A M, Westmoreland D, Bagg J. Seroepidemiological study of respiratory virus infections among dental surgeons. Br Dent J 1994; 176: 262-265.

2. Wölfel R, Corman V M, Guggemos W et al. Virological assessment of hospitalized patients with COVID-2019. Nature 2020; 581: 465-469.

3. Bullard J, Dust K, Funk D et al. Predicting infectious SARS-CoV-2 from diagnostic samples. Clin Infect Dis 2020; DOI: 10.1093/cid/ciaa638

4. La Scola B, Le Bideau M, Andreani J et al. Viral RNA load as determined by cell culture as a management tool for discharge of SARS-CoV-2 patients from infectious disease wards. Eur J Clin Microbiol Infect Dis 2020; 39: 1059-1061.

5. He X, Lau E H Y, Wu P et al. Temporal dynamics in viral shedding and transmissibility of COVID-19. Nat Med 2020; 26: 672-675.

6. Hao-Yuan Cheng HY, Jian SW, Liu DP et al. Contact tracing assessment of COVID-19 transmission dynamics in Taiwan and risk at different exposure periods before and after symptom onset. JAMA Intern Med 2020; 180: 1156-1163.

7. Carmo A, Pereira-Vaz J, Mota V et al. Clearance and persistence of SARS-CoV-2 RNA in patients with COVID-19. J Med Virol 2020; DOI: 10.1002/jmv. 26103.

8. Jones T C, Mühlemann B, Veith T et al. An analysis of SARS-CoV-2 viral load by patient age. 2020. Available online at https://www.medrxiv.org/ content/10.1101/2020.06.08.20125484v1 (accessed September 2020).

9. Decker A, Welzel M, Laubner K et al. Prolonged SARSCOV2 shedding and mild course of COVID-19 in a patient after recent heart transplantation. $A m$ J Transplant 2020; DOI: 10.1111/ajt.16133.

10. Long Q, Tang X, Shi Q et al. Clinical and immunological assessment of asymptomatic SARS-COV-2 infections. Nat Med 2020; 26: 1200-1204.

11. Du Z, Zhu F, Guo F, Yang B, Wang T. Detection of antibodies against SARS-CoV-2 in patients with COVID-19. J Med Virol 2020; DOI: 10.1002/jmv.25820.

12. Qu J, Wu C, Li X et al. Profile of IgG and IgM antibodies against Severe Acute Respiratory Syndrome coronavirus 2 (SARS-CoV-2). Clin Infect Dis 2020; DOI: 10.1093/cid/ciaa489.

13. Markland E, Leach S, Axelsson $\mathrm{H}$ et al. Serum-IgG responses to SARS-CoV-2 after mild and severe COVID19 infection and analysis of IgG non-responders. 2020. Available online at https://www.medrxiv.org/ content/10.1101/2020.07.11.20151324v1 (accessed September 2020).

14. Grifoni A, Weiskopf D, Ramirez S I et al. Targets of T cell responses to SARS-CoV-2 coronavirus in humans with COVID-19 disease and unexposed individuals. Cell 2020; DOI: 10.1016/j.cell.2020.05.015.

15. Van Elslande J, Houben E, Depypere M et al. Diagnostic performance of seven rapid IgG/IgM antibody tests and the Euroimmun IgA/IgG ELISA in COVID-19 patients. Clin Microbiol Infect 2020; 26: 1082-1087.

16. Lassaunière R, Frische $A$, Harboe $Z$ B et al. Evaluation of nine commercial SARS-CoV-2 immunoassays. 2020. Available online at https://www.medrxiv.org/ content/10.1101/2020.04.09.20056325v1 (accessed September 2020).

17. Kohmer N, Westhaus $S$, Rühl C, Ciesek S, Rabenau H F. Clinical performance of different SARS-CoV-2 IgG antibody tests. J Med Virol 2020; DOI: 10.1002/ jmv.26145. 
18. Ai T, Yang Z, Hou H et al. Correlation of chest CT and RTPCR testing in coronavirus disease 2019 (COVID-19) in China: A report of 1014 cases. Radiology 2020; DOI: 10.1148/radiol.2020200642

19. Danh K, Karp D G, Robinson P V et al. Detection of SARS-CoV-2 neutralizing antibodies with a cell-free PCR assay. 2020. Available online at https://www. medrxiv.org/content/10.1101/2020.05.28.20105692v1 (accessed September 2020).

20. Rasheed A M, Fatak D F, Hashim H A et al. The therapeutic potential of convalescent plasma therapy on treating critically-ill COVID-19 patients residing in respiratory care units in hospitals in Baghdad, Iraq. Infez Med 2020; 28: 357-366.

21. Joyner M J, Senefeld J W, Klassen S A et al. Effect of Convalescent Plasma on Mortality among Hospitalized Patients with COVID-19: Initial Three-Month Experience. 2020. Available online at https://www. medrxiv.org/content/10.1101/2020.08.12.20169359v1 (accessed September 2020).

22. Deng W, Bao L, Liu J et al. Primary exposure to SARS-CoV-2 protects against reinfection in rhesus macaques. Science 2020; DOI: 10.1126/science. abc5343.

23. Peng Y, Mentzer A J, Liu G et al. Broad and strong Memory CD4 + and CD8 + T cells induced by SARS-CoV-2 in UK convalescent COVID-19 patients. 2020. Available online at https://www.biorxiv.org/ content/10.1101/2020.06.05.134551v1 (accessed September 2020).

24. Seow J, Graham C, Merrick B et al. Longitudinal evaluation and decline of antibody responses in SARS-CoV-2 infection. 2020. Available online at https://www.medrxiv.org/ content/10.1101/2020.07.09.20148429v1 (accessed September 2020)

25. Porte L, Legarraga P, Vollrath V et al. Evaluation of a novel antigen-based rapid detection test for the diagnosis of SARS-CoV-2 in respiratory samples. Int J Infect Dis 2020; 99: 328-333.

26. Paltiel D A, Walensky R P. Screening to prevent SARS-CoV-2 outbreaks: Saliva-based antigen testing is better than the PCR swab. 2020. Available at https://www.healthaffairs.org/do/10.1377/ hblog20200909.430047/full/ (accessed September 2020).

27. UK Biobank. UK Biobank COVID-19 antibody study: latest updates. 2020. Available at https://www. ukbiobank.ac.uk/2020/07/uk-biobank-covid-19antibody-study-latest-updates/ (accessed September 2020).

28. Public Health Scotland. Enhanced surveillance of COVID-19 in Scotland - Population-based seroprevalence surveillance: A Management Information Statistics publication for Scotland. 2020. Available at https://beta.isdscotland.org/findpublications-and-data/population-health/covid-19/ enhanced-surveillance-of-covid-19-in-scotland/ (accessed September 2020).

29. Ward H, Christina J Atchison, Matthew Whitaker et al. Antibody prevalence for SARS-CoV-2 in England following first peak of the pandemic: REACT2 study in 100,000 adults. 2020.

Available online at https://www.medrxiv.org/ content/10.1101/2020.08.12.20173690v2 (accessed September 2020). 